\title{
Magnetic phase separation in ordered alloys
}

\author{
Jordi Marcos, Eduard Vives, and Teresa Castán \\ Departament d'Estructura i Constituents de la Matèria, Facultat de Física, Universitat de Barcelona, Diagonal 647 , \\ E-08028 Barcelona, Catalonia, Spain
}

(Received 20 September 2000; published 23 May 2001)

\begin{abstract}
We present a lattice model to study the equilibrium phase diagram of ordered alloys with one magnetic component that exhibits a low temperature phase separation between paramagnetic and ferromagnetic phases. The model is constructed from the experimental facts observed in $\mathrm{Cu}_{3-x} \mathrm{AlMn}_{x}$ and it includes coupling between configurational and magnetic degrees of freedom that are appropriate for reproducing the low temperature miscibility gap. The essential ingredient for the occurrence of such a coexistence region is the development of ferromagnetic order induced by the long-range atomic order of the magnetic component. A comparative study of both mean-field and Monte Carlo solutions is presented. Moreover, the model may enable the study of the structure of ferromagnetic domains embedded in the nonmagnetic matrix. This is relevant in relation to phenomena such as magnetoresistance and paramagnetism.
\end{abstract}

DOI: $10.1103 /$ PhysRevB.63.224418

PACS number(s): 75.40.Mg, 64.60.Cn, 64.75.+g

\section{INTRODUCTION}

Recently, renewed interest has been addressed to ferromagnetic ordered alloys. This is because of the unique properties arising from the interplay between elasticity, magnetism, and (configurational) atomic order. From the point of view of applications, the development of actuator materials having very large magnetostrains ${ }^{1}$ is particularly interesting. Also relevant is the possibility of having superparamagnetism $^{2}$ and giant magnetoresistance, ${ }^{3}$ both associated with coexistence of magnetic domains (large magnetic particles) embedded in a nonmagnetic matrix. This mixed phase has been observed, for instance, in the $\mathrm{Cu}_{3-x} \mathrm{AlMn}_{x}$ Heusler alloy.

The Heusler alloys are ternary intermetallic compounds with the composition $X_{2} Y Z$ and a low temperature $L 2_{1}$ structure. At high temperatures the stable phase corresponds to a disordered bcc lattice, also called the $A_{2}$ phase, which undergoes a two-stage disorder-order transition $A_{2} \rightarrow B_{2}$ $\rightarrow L 2_{1}$, as the temperature is decreased. Especially interesting are the Mn-based Heusler alloys ${ }^{4-7}$ which exhibit a magnetic moment approximately located on the Mn atoms. ${ }^{8}$ Among them, the most extensively studied are the $\mathrm{Ni}_{2} \mathrm{GaMn}$ (Refs. 9 and 10) and the $\mathrm{Cu}_{2}$ AlMn (Refs. 11-17) alloys. In both cases, the $L 2_{1}$ phase is ferromagnetic but the $B_{2}$ phase is paramagnetic. This close relation between atomic order and magnetic properties has been known to scientists for many years. ${ }^{18}$ Additionally, these alloys exhibit shapememory effects, intimately related to the structural transition, of the martensitic type, ${ }^{19}$ undergone at low temperatures. It has been suggested that the control of shape-memory properties by application of an external magnetic field is a principle for operation of the recently developed actuator materials. ${ }^{20,21}$

In $\mathrm{Cu}-\mathrm{Al}-\mathrm{Mn}$ the martensitic transition exists ${ }^{14}$ only for compositions that are very far from the stoichiometry $\left(\mathrm{Cu}_{2} \mathrm{AlMn}\right)$ where the $L 2_{1}$ ordered phase is paramagnetic. ${ }^{22}$ Nevertheless, the influence of magnetism coming from $\mathrm{Mn}$ has been revealed in several experiments. ${ }^{23}$ As well as the phase transitions mentioned above, the system exhibits, at low temperatures, a spinodal decomposition along the line $\mathrm{Cu}_{3} \mathrm{Al}-\mathrm{Cu}_{2} \mathrm{AlMn}{ }^{11,12,24} \mathrm{We}$ will center our attention on this two-phase region and denote the $\mathrm{Cu}$-rich portion of the phase diagram of interest in this paper by $\mathrm{Cu}_{3-x} \mathrm{AlMn}_{x}$, with 0 $\leqslant x \leqslant 1$. In Fig. 1 we show schematically the corresponding phase diagram as it is obtained from experiment. The continuous lines are drawn from the data in Ref. 24, whereas the points at $x=0$ and $x=0.28$ are from Refs. 25 and 26, respectively.

The low temperature ordered structures for the limiting values of $x$ are different. The $\mathrm{Cu}_{3} \mathrm{Al}$ binary alloy is $\mathrm{DO}_{3}$ at low temperatures, ${ }^{27}$ whereas $\mathrm{Cu}_{2} \mathrm{AlMn}$ is $L 2_{1}$ and ferromagnetic, with a relatively high Curie temperature $(\sim 630 \mathrm{~K}) .{ }^{28}$ The ferromagnetism of the $L 2_{1}$ phase appears as a consequence of the atomic ordering of the Mn atoms. In this sense it is known that properties such as the saturation magnetic moment depend on the degree of order of the Mn atoms. ${ }^{29}$ It then naturally follows that the absence of magnetism (longrange magnetic order) either in the high temperature $B_{2}$ phase or in the low temperature phase $\left(D O_{3}\right.$ or $\left.L 2_{1}\right)$, for small values of $x$ might well be related to the tendency for the Mn atoms to distribute themselves randomly at different lattice sites. On the other hand, by increasing the amount of

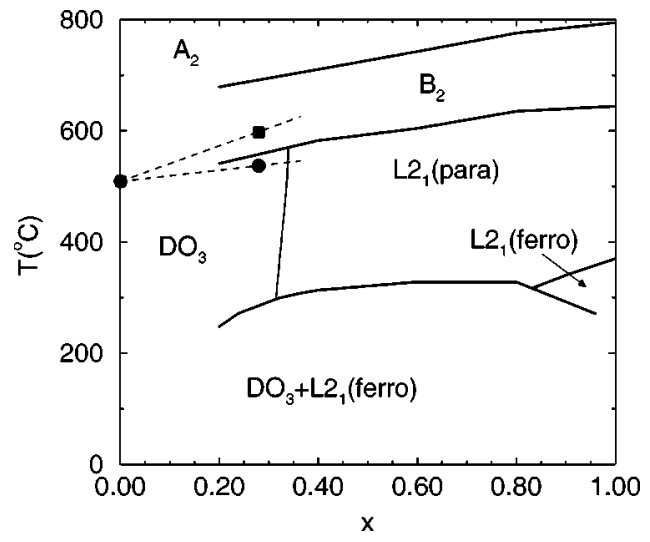

FIG. 1. Approximate experimental phase diagram of $\mathrm{Cu}_{3-x} \mathrm{AlMn}_{x}$ from Refs. 24, 25, and 26. 
$\mathrm{Mn}$, for instance in the $\mathrm{Cu}_{3} \mathrm{AlMn}_{2}$ alloy, the resulting magnetic interaction becomes antiferromagnetic. ${ }^{29}$ Such different magnetic behavior may be understood in terms of the oscillatory Ruderman-Kittel-Kasuya-Yosida interaction between the magnetic moments of the $\mathrm{Mn}$ atoms. ${ }^{30,17,31}$

The phase separation or miscibility gap in $\mathrm{Cu}_{2} \mathrm{AlMn}$ occurs at temperatures below $\sim 600 \mathrm{~K}$ (see Fig. 1), ${ }^{12}$ and gives rise to a coexistence region between a nonmagnetic phase and a ferromagnetic phase, with low and high Mn content, respectively. The occurrence of superparamagnetism ${ }^{2}$ or magnetoresistance ${ }^{3}$ is directly related to the existence of magnetic clusters (stable $L 2_{1}$ domains) immersed in the nonmagnetic $\left(\mathrm{DO}_{3}\right)$ matrix. Some aspects of this phase diagram are not totally clear. First is the persistence of a stable $\mathrm{DO}_{3}$ phase for small values of $x$. Kainuma et al. ${ }^{24}$ by using x-ray diffraction measurements, have detected an abrupt change in the intensity of the superstructure peaks at $x \simeq 0.32$. It should be mentioned that this effect was not found in other earlier studies. ${ }^{11}$ Other important information, not yet available, refers to the different atomic distributions for the nonstoichiometric $L 2_{1}$ structure. Some assumptions on this matter will be required in order to perform a theoretical study. Other aspects that need to be discussed refer to the characteristics of the coexisting phases. They will depend on the location of the $\mathrm{DO}_{3}-L 2_{1}$ (according to the results in Ref. 24) and magnetic transition lines with respect to the coexistence line. More precisely, depending on the temperatures at which such interphases end on the coexistence line, the phases may be different in atomic order $\left(\mathrm{DO}_{3}, \mathrm{~L}_{2}\right)$ or/and magnetic order (ferromagnetic, paramagnetic). In this sense, even the coexistence of two different paramagnetic $L 2_{1}$ and $L 2_{1}^{\prime}$ phases (upper part of the miscibility gap in Fig. 1), with a very similar content of $\mathrm{Mn}$, has been suggested. ${ }^{24}$

In this paper we present a lattice model able to reproduce the main features of the equilibrium phase diagram in this two-phase region. The details of the model will be derived from a microscopic description of the atomic and magnetic properties of $\mathrm{Cu}_{3-x} \mathrm{AlMn}_{x}$ alloys. Nevertheless, it can be applied to other systems. For practical reasons we will make several hypotheses which in some cases are not totally justified a priori but only later from agreement of the results obtained with experimental data. This agreement is indicative that the model captures the essential physics and provides a starting point for future more exhaustive studies. The model is a projection of a ternary alloy onto a binary system, when one of the species is magnetic. It is constructed on the basis that the main physics of the phenomenon lies on the atomic ordering of the magnetic component, which, moreover, is taken to be always the less abundant one. The effective Hamiltonian accounts for a purely configurational ordering energy between first neighboring pairs so that at low temperatures the magnetic atoms tend to be second neighbors. Then a simple ferromagnetic pair interaction between next-nearest neighbors is enough to give rise to a low temperature phase separation between a nonmagnetic phase and a ferromagnetic phase that, moreover, may have different ordered structures.

It has been suggested ${ }^{24}$ that the occurrence of the twophase region in $\mathrm{Cu}_{3-x} \mathrm{AlMn}_{x}$ cannot be attributed to either chemical (configurational) or magnetic ordering. In this work, we use a very simple microscopic model to show that the coupling between the atomic (configurational) and magnetic orderings is sufficient to give rise to a decomposition between two phases at low temperatures. This coupling operates in such a way that, as the atomic ordering develops, the (indirect) exchange interactions between the atomic moments of the magnetic particles produce long-range ferromagnetic order.

The remainder of the paper is organized as follows. In Sec. II we introduce the model. Section III is devoted to its mean-field solution. In order to better understand the nature of the different phases and the behavior of several measurable quantities we also solve the model by using Monte Carlo numerical simulations. This is presented in Sec. IV. Finally in Sec. V we summarize and conclude.

\section{MODEL}

In the present study, our main goal is to understand the formation of the miscibility gap in $\mathrm{Cu}_{3-x} \mathrm{AlMn}_{x}$ along the line $0 \leqslant x \leqslant 1$. The complexity inherent in the description of a magnetic ternary alloy has led us to make simplifications that we shall discuss in this section. Indeed, the quest for reasonable simplifications becomes compulsory in order to perform the Monte Carlo numerical simulations. Although the inclusion of too many ingredients (and thus free parameters) in the model may lead to a better fit of the available data (in our case scarce), it may hide the understanding of the relevant physical mechanism underlying the phase diagram properties, which we believe is the coupling between the long-range configurational (chemical) ordering and the magnetism of the $\mathrm{Mn}$ atoms.

The equilibrium structure of $\mathrm{Cu}_{3-x} \mathrm{AlMn}_{x}$ can be described as an underlying bcc structure formed by the superposition of four interpenetrated fcc sublattices, named $\alpha, \beta, \gamma$, and $\delta$ [see Fig. 2(a)]. In order to describe the different phases of the system it is convenient to specify the occupation probabilities $p_{X}^{\Sigma}$ of the different species $X$ $(=\mathrm{Cu}, \mathrm{Al}, \mathrm{Mn})$ in the four different sublattices $\Sigma$ $(=\alpha, \beta, \gamma, \delta)$. Tables I and II summarize the occupation probabilities for the limiting $\mathrm{DO}_{3}(x=0)$ and $L 2_{1} \quad(x=1)$ stoichiometric phases. For intermediate values of $x$ a more elaborate discussion is required.

We start with the region corresponding to small values of $x, x \geq 0$. Recently ${ }^{24} \mathrm{x}$-ray diffraction experiments showed that the $\mathrm{DO}_{3}$ structure persists above the coexistence region for values of $x$ up to 0.32 . In other words, the addition of a small amount of $\mathrm{Mn}$ does not break the symmetry $p_{X}^{\gamma}=p_{X}^{\delta}$

TABLE I. Occupation probabilities for the stoichiometric $\mathrm{DO}_{3}$ structure of $\mathrm{Cu}_{3} \mathrm{Al}$.

\begin{tabular}{lllll}
\hline \hline & $\alpha$ & $\beta$ & $\gamma$ & $\delta$ \\
\hline $\mathrm{Cu}$ & 0 & 1 & 1 & 1 \\
$\mathrm{Al}$ & 1 & 0 & 0 & 0 \\
$\mathrm{Mn}$ & 0 & 0 & 0 & 0 \\
\hline \hline
\end{tabular}


(a)

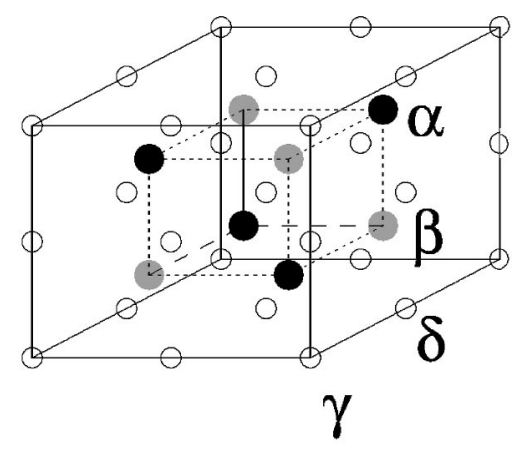

(b)

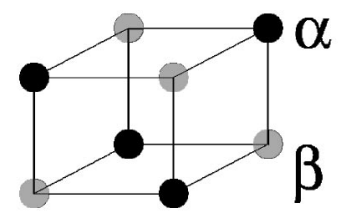

FIG. 2. (a) Structure of the $L 2_{1}$ phase of $\mathrm{Cu}_{2} \mathrm{AlMn}$ indicating the $\alpha, \beta, \gamma$, and $\delta$ sublattices. (b) Cell used for the present model.

$=p_{X}^{\alpha}$. This is an (a priori) unexpected result, given the different atomic environments of these sublattices in the $\mathrm{DO}_{3}$ phase. In any case, it seems clear that entropy plays a very important role in the stability of this homogeneous $\mathrm{DO}_{3}$ phase. From Fig. 1 it follows that for $x \gtrsim 0$ the stability is extended to higher temperatures as the value of $x$ increases. A natural hypothesis is, therefore, to assume that (for low values of $x$ ) the $\mathrm{Mn}$ atoms behave as impurities that are randomly distributed on the four different sublattices. The corresponding occupation probabilities $p_{X}^{\Sigma}$ are indicated in Table III.

In the $x \lesssim 1$ region the stable phase is of the $L 2_{1}$ type. There are several atomic distributions that are compatible with the symmetry $p_{X}^{\gamma}=p_{X}^{\delta} \neq p_{X}^{\alpha} \neq p_{X}^{\beta}$. Table IV displays the occupation probabilities in the most straightforward case for which the Mn concentrates in a unique sublattice. Alternatively, in a more general way, one might write the occupation probabilities (Table $\mathrm{V}$ ) in terms of a free parameter $\lambda(0 \leqslant \lambda \leqslant 1)$. Notice that these atomic distributions account for a continuous change from the $\mathrm{DO}_{3}$ phase $(\lambda=0)$ to the $L 2{ }_{1}$ phase $(\lambda>0)$.

The next step is to introduce the two major simplifications of the model.

(1) The structures described in Tables I-V have the exis-

TABLE II. Occupation probabilities for the stoichiometric $L 2_{1}$ structure of $\mathrm{Cu}_{2} \mathrm{AlMn}$.

\begin{tabular}{lllll}
\hline \hline & $\alpha$ & $\beta$ & $\gamma$ & $\delta$ \\
\hline $\mathrm{Cu}$ & 0 & 0 & 1 & 1 \\
$\mathrm{Al}$ & 1 & 0 & 0 & 0 \\
$\mathrm{Mn}$ & 0 & 1 & 0 & 0 \\
\hline \hline
\end{tabular}

TABLE III. Guessed occupation probabilities for the nonstoichiometric $\mathrm{DO}_{3}$ structure with composition $x \gtrsim 0$.

\begin{tabular}{ccccc}
\hline \hline & $\alpha$ & $\beta$ & $\gamma$ & $\delta$ \\
\hline $\mathrm{Cu}$ & 0 & $1-\frac{x}{3}$ & $1-\frac{x}{3}$ & $1-\frac{x}{3}$ \\
$\mathrm{Al}$ & $1-\frac{x}{4}$ & $\frac{x}{12}$ & $\frac{x}{12}$ & $\frac{x}{12}$ \\
$\mathrm{Mn}$ & $\frac{x}{4}$ & $\frac{x}{4}$ & $\frac{x}{4}$ & $\frac{x}{4}$ \\
\hline \hline
\end{tabular}

tence of two nearest-neighbor sublattices ( $\gamma$ and $\delta$ ) in common which contain most of the $\mathrm{Cu}$ atoms and have identical occupation probabilities: $p_{X}^{\gamma}=p_{X}^{\delta}, \forall X$. Experimentally, this symmetry with respect to the $\gamma$ and $\delta$ sublattices seems to be satisfied for any concentration and temperature range. From now on we forget about them and concentrate on the atomic distribution behavior on the other two remaining sublattices, motivated by the feeling that the breaking down of the $\alpha-\beta$ symmetry is crucial in the ordering of the Mn atoms at low temperatures. This, of course, will restrict the validity of our study to temperatures below the $\mathrm{B}_{2}-\mathrm{DO}_{3}$ transition, which is precisely the region of interest here. Therefore, the model will be defined on a simple cubic lattice divided into two sublattices, $\alpha$ and $\beta$, as illustrated in Fig. 2(b).

(2) Continuing with our assumption that the main physics lies in the atomic ordering of the $\mathrm{Mn}$ atoms, we shall proceed further by distinguishing between magnetic and nonmagnetic atoms only. In our binary alloy model $A_{1-c} B_{c}$, the nonmagnetic species $A$ stands for either $\mathrm{Cu}$ or $\mathrm{Al}$, whereas the magnetic species $B$ stands for $\mathrm{Mn}$ and the composition is restricted to $c<0.50$. The behavior of the $B$ atoms on sublattices $\alpha$ and $\beta$ can be regarded as a simple orderdisorder transition. For small values of $c$ both sublattices are equally populated by $B$ atoms (behaving as impurities) while for larger values of $c B$ atoms occupy preferably one of the two sublattices. Moreover, this behavior depends on temperature. As regards the configurational ordering, the model gives rise to two phases only: disordered and ordered, corresponding to low $\left(\mathrm{DO}_{3}\right)$ and high $\left(\mathrm{L2}_{1}\right)$ content of the magnetic species, respectively. Keeping this correspondence in mind, in what follows we shall use the simplified notation $D$ (disordered) and $O$ (ordered).

We notice that the quantitative study of properties such as the magnetization, susceptibility, or other properties related to magnetism (magnetoresistance, etc.) is not our goal here. Rather, we shall focus on how the development of longrange ferromagnetic order (resulting from the interplay with the atomic order) determines the phase diagram at low tem-

TABLE IV. Simplest occupation probabilities for the nonstoichiometric $L 21_{1}$ structure with composition $x \leqq 1$.

\begin{tabular}{lcccc}
\hline \hline & $\alpha$ & $\beta$ & $\gamma$ & $\delta$ \\
\hline $\mathrm{Cu}$ & 0 & $1-x$ & 1 & 1 \\
$\mathrm{Al}$ & 1 & 0 & 0 & 0 \\
$\mathrm{Mn}$ & 0 & $x$ & 0 & 0 \\
\hline \hline
\end{tabular}


TABLE V. Guessed occupation probabilities for the nonstoichiometric $\mathrm{Cu}_{3-x} \mathrm{AlMn}_{x}$ alloys that include the $\mathrm{DO}_{3}$ structure $(\lambda=0)$ and the $L 2_{1}$ structure $(\lambda \neq 0)$.

\begin{tabular}{ccccc}
\hline \hline & $\alpha$ & $\beta$ & $\gamma$ & $\delta$ \\
\hline $\mathrm{Cu}$ & 0 & $1-\frac{x}{3}(1+2 \lambda)$ & $1-\frac{x}{3}(1-\lambda)$ & $1-\frac{x}{3}(1-\lambda)$ \\
$\mathrm{Al}$ & $1-\frac{x}{4}(1-\lambda)$ & $\frac{x}{12}(1-\lambda)$ & $\frac{x}{12}(1-\lambda)$ & $\frac{x}{12}(1-\lambda)$ \\
$\mathrm{Mn}$ & $\frac{x}{4}(1-\lambda)$ & $\frac{x}{4}(1+3 \lambda)$ & $\frac{x}{4}(1-\lambda)$ & $\frac{x}{4}(1-\lambda)$ \\
\hline \hline
\end{tabular}

peratures as a function of the content of the magnetic species $c$. In terms of the model description, this can be achieved by considering localized Ising-like spin variables $s_{i}= \pm 1$ associated with each $B$ atom.

We start from the following pair-interaction effective Hamiltonian:

$$
\begin{aligned}
\mathcal{H}= & \mathcal{H}^{c}+\mathcal{H}^{m} \\
= & \sum_{k=1}^{w}\left[N_{A A}^{k} \epsilon_{A A}^{k}+N_{A B}^{k} \epsilon_{A B}^{k}+N_{B B}^{k} \epsilon_{B B}^{k}\right] \\
& +\sum_{k=1}^{w}\left[N_{B^{+} B^{+}}^{k} \epsilon_{++}^{k}+N_{B^{+} B^{-}}^{k} \epsilon_{+-}^{k}+N_{B^{-} B^{-}}^{k} \epsilon_{--}^{k}\right],
\end{aligned}
$$

where $\mathcal{H}^{c}$ and $\mathcal{H}^{m}$ are the configurational and magnetic energy contributions, respectively. The summation is performed over the different $k$-nearest-neighbor shells (up to $k$ $=w), N_{X X}^{k}$ is the number of $k$ th nearest-neighbor $X$ - $X$ pairs, and $\epsilon_{X X}^{k}$ are their corresponding pair-interaction energies. Note that the magnetic contribution involves only $B$ atoms. We have indicated by $B^{+}$and $B^{-}$the two possible magnetic states. To preserve the symmetry under exchange of the + and - magnetic states we take $\epsilon_{++}^{k}=\epsilon_{--}^{k} \forall k$.

Following standard procedures, we write Hamiltonian (1) in terms of Ising-like variables defined at each lattice site. Let us index the sites of the cubic lattice by $i$ $=1, \ldots, N(N=L \times L \times L)$. At each lattice site $i$ we define the following two coupled two-state variables $\sigma_{i}$ and $S_{i}$. The variable $\sigma_{i}=+1,-1$ represents the nonmagnetic and magnetic species $\left(A\right.$ and $B$ ), respectively; then, provided $\sigma_{i}$ $=-1$, we define $S_{i}=+1,-1$ describing the two possible magnetic states of each $B$ atom.

Considering interactions up to next-nearest neighbors $(w$ $=2$ ), the configurational energy term in Eq. (1) can be written, neglecting constant terms, as

$$
\mathcal{H}^{c}=J_{1}^{c} \sum_{i j}^{\mathrm{NN}} \sigma_{i} \sigma_{j}+J_{2}^{c} \sum_{i j}^{\mathrm{NNN}} \sigma_{i} \sigma_{j}+E^{c} \sum_{i=1}^{N} \sigma_{i},
$$

where the first two summations are extended to nearest neighbors (NN) and next-nearest neighbors (NNN), respectively, and the Hamiltonian parameters are

$$
J_{1}^{c}=\frac{\epsilon_{A A}^{1}+\epsilon_{B B}^{1}-2 \epsilon_{A B}^{1}}{4},
$$

$$
J_{2}^{c}=\frac{\epsilon_{A A}^{2}+\epsilon_{B B}^{2}-2 \epsilon_{A B}^{2}}{4},
$$

and

$$
E^{c}=\frac{z_{1}}{2}\left(\epsilon_{A A}^{1}-\epsilon_{B B}^{1}\right)+\frac{z_{2}}{2}\left(\epsilon_{A A}^{2}-\epsilon_{B B}^{2}\right),
$$

where $z_{1}=6$ and $z_{2}=12$ are the numbers of NN's and NNN's of each lattice site. In the canonical ensemble, the last term in Eq. (2) is just a simple energy shift which depends on the alloy concentration. The magnetic energy term can be rewritten as

$$
\begin{aligned}
\mathcal{H}^{m}= & J_{1}^{m} \sum_{i j}^{\mathrm{NN}} \frac{1-\sigma_{i}}{2} \frac{1-\sigma_{j}}{2} S_{i} S_{j}+J_{2}^{m} \sum_{i j}^{\mathrm{NNN}} \frac{1-\sigma_{i}}{2} \frac{1-\sigma_{j}}{2} S_{i} S_{j} \\
& +4 K_{1}^{m} \sum_{i j} \frac{1-\sigma_{i}}{2} \frac{1-\sigma_{j}}{2}+4 K_{2}^{m} \sum_{i j}^{N N N} \frac{1-\sigma_{i}}{2} \frac{1-\sigma_{j}}{2}
\end{aligned}
$$

where

$$
\begin{gathered}
J_{1}^{m}=\frac{\epsilon_{++}^{1}-\epsilon_{+-}^{1}}{2}, \quad J_{2}^{m}=\frac{\epsilon_{++}^{2}-\epsilon_{+-}^{2}}{2}, \\
K_{1}^{m}=z_{1} \frac{\epsilon_{++}^{1}+\epsilon_{+-}^{1}}{8}, \quad K_{2}^{m}=z_{2} \frac{\epsilon_{++}^{2}+\epsilon_{+-}^{2}}{8} .
\end{gathered}
$$

We note that the last two terms in Eq. (6) do not depend on the magnetic variables $\left\{S_{i}\right\}$. Expanding the different contributions in Eq. (6) and ignoring constant terms, the Hamiltonian becomes

$$
\begin{aligned}
\mathcal{H}= & \left(J_{1}^{c}+K_{1}^{m}\right) \sum_{i j}^{\mathrm{NN}} \sigma_{i} \sigma_{j}+\left(J_{2}^{c}+K_{2}^{m}\right) \sum_{i j}^{\mathrm{NNN}} \sigma_{i} \sigma_{j} \\
& +J_{1}^{m} \sum_{i j}^{\mathrm{NN}} \frac{1-\sigma_{i}}{2} \frac{1-\sigma_{j}}{2} S_{i} S_{j}+J_{2}^{m} \sum_{i j}^{\mathrm{NNN}} \frac{1-\sigma_{i}}{2} \frac{1-\sigma_{j}}{2} S_{i} S_{j} .
\end{aligned}
$$

The superscripts in the model parameters denote its configurational $\left(^{c}\right)$ or magnetic $\left({ }^{m}\right)$ origin, whereas the subscripts mean first- $\left({ }_{1}\right)$ or second- $\left({ }_{2}\right)$ neighbor interactions. In order to reduce the number of free model parameters we set $J_{1}^{m}$ $=0$. Indeed, the NN magnetic interaction between $B-B$ pairs is not essential for our present purposes since we restrict ourselves to the case in which the ferromagnetism develops in the configurationally ordered phase. Furthermore, by using reduced energy units $\mathcal{H}^{*}=\mathcal{H} /\left(J_{1}^{c}+K_{1}^{m}\right)$, we get the following minimal model Hamiltonian:

$$
\mathcal{H}^{*}=\sum_{i j}^{\mathrm{NN}} \sigma_{i} \sigma_{j}-K^{*} \sum_{i j}^{\mathrm{NNN}} \sigma_{i} \sigma_{j}-J_{2}^{*} \sum_{i j}^{\mathrm{NNN}} \frac{1-\sigma_{i}}{2} \frac{1-\sigma_{j}}{2} S_{i} S_{j},
$$

where the parameters are 


$$
K^{*}=-\frac{J_{2}^{c}+K_{2}^{m}}{J_{1}^{c}+K_{1}^{m}}
$$

which measures the ordering energy between secondneighbor pairs, either $A-A, B-B$, or $A-B$, independently of the magnetic state of atom $B$, and

$$
J_{2}^{*}=-\frac{J_{2}^{m}}{J_{1}^{c}+K_{1}^{m}}
$$

which accounts for the ferromagnetic interaction between second-neighbor $B-B$ pairs.

\section{MEAN-FIELD SOLUTION}

This section is devoted to the solution of the model introduced previously for the $A_{1-c} B_{c}$ binary alloy by using standard mean-field techniques based on the Bragg-Williams approximation. We denote the occupation numbers for each component $\left(X=A, B^{+}, B^{-}\right)$in each sublattice $(\Sigma=\alpha, \beta)$ by $N_{X}^{\Sigma}$ and consider the following order parameters:

$$
\begin{gathered}
c=\frac{N_{B}^{\alpha+}+N_{B}^{\alpha-}+N_{B}^{\beta+}+N_{B}^{\beta-}}{N}, \\
\eta=2 \frac{N_{B}^{\alpha+}+N_{B}^{\alpha-}-N_{B}^{\beta+}-N_{B}^{\beta-}}{N}, \\
m=\frac{N_{B}^{\alpha+}+N_{B}^{\beta+}-N_{B}^{\alpha-}-N_{B}^{\beta-}}{N},
\end{gathered}
$$

where $c(0<c<0.5)$ is the molar fraction of the magnetic species, $\eta(0<\eta<2 c)$ is the atomic order parameter, and $m$ $(0<m<2 c)$ measures the magnetization of the system. Using standard procedures, in the grand canonical formulation, we obtain the following expression for the internal energy:

$$
\begin{aligned}
E= & N J_{1}\left[\frac{1}{2}(1-2 c)^{2}\left(1-K^{*} \frac{z_{2}}{z_{1}}\right)-\frac{\eta^{2}}{2}\left(1+K^{*} \frac{z_{2}}{z_{1}}\right)\right. \\
& \left.+\mu^{*}(1-2 c)-J_{2}^{*} \frac{z_{2}}{z_{1}} m^{2}\right],
\end{aligned}
$$

where $J=J_{1}^{c}+K_{1}^{m}$ and $\mu^{*}$ is the chemical potential difference between the two species. The corresponding entropy is given by

$$
S=k_{B} \ln \left[\frac{N^{\alpha} !}{N_{A}^{\alpha} ! N_{B^{+}}^{\alpha} ! N_{B^{-}}^{\alpha} !}\right]\left[\frac{N^{\beta} !}{N_{A}^{\beta} ! N_{B^{+}}^{\beta} ! N_{B^{-}}^{\beta} !}\right]
$$

Expressions (16) and (17) produce the following free energy:
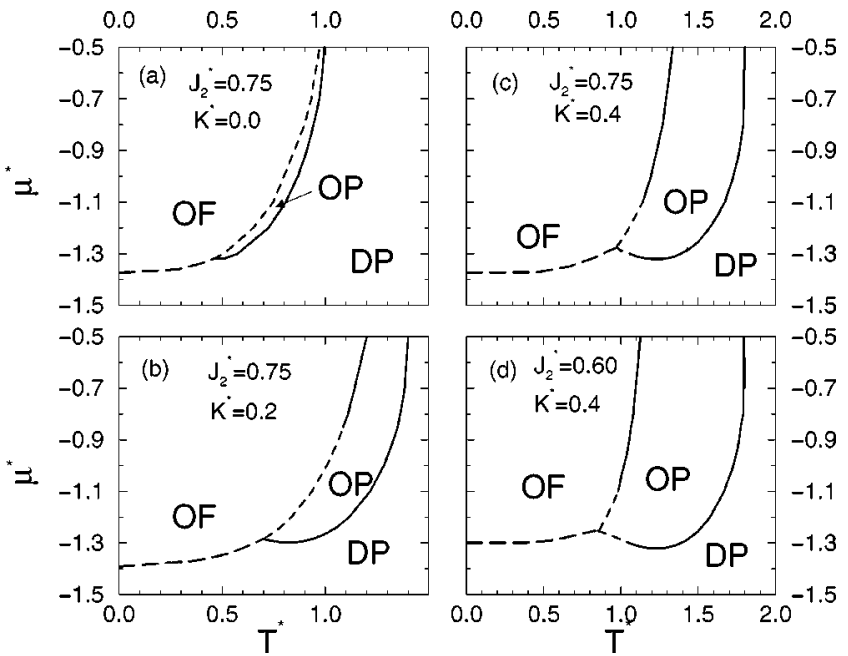

FIG. 3. Mean-field $\mu^{*}-T^{*}$ diagrams for different values of model parameters $J_{2}^{*}$ and $K^{*}$, indicating the disorderedparamagnetic phase (DP), the ordered-paramagnetic phase (OP), and the ordered-ferromagnetic phase (OF). Dashed lines indicate first-order phase transitions while continuous lines stand for continuous phase transitions.

$$
\begin{aligned}
\mathcal{F}^{*}= & \frac{F}{N J z_{1}} \\
= & \frac{E}{N J z_{1}}-\left(\frac{T}{J z_{1}}\right)\left(\frac{S}{N}\right) \\
= & {\left[\frac{1}{2}(1-2 c)^{2}\left(1-K^{*} \frac{z_{2}}{z_{1}}\right)\right.} \\
& \left.-\frac{\eta^{2}}{2}\left(1+K^{*} \frac{z_{2}}{z_{1}}\right)+\mu^{*}(1-2 c)-J_{2}^{*} \frac{z_{2}}{z_{1}} m^{2}\right] \\
& +\frac{T^{*}}{4}\left[2\left(1-c-\frac{\eta}{2}\right) \ln \left(1-c-\frac{\eta}{2}\right)+2\left(1-c+\frac{\eta}{2}\right)\right. \\
& \times \ln \left(1-c+\frac{\eta}{2}\right)+\left(c+\frac{\eta}{2}+2 m\right) \ln \left(c+\frac{\eta}{2}+2 m\right) \\
& +\left(c+\frac{\eta}{2}-2 m\right) \ln \left(c+\frac{\eta}{2}-2 m\right) \\
& \left.+2\left(c-\frac{\eta}{2}\right) \ln \left(c-\frac{\eta}{2}\right)-4 c \ln 2\right],
\end{aligned}
$$

with $T^{*}=T k_{B} / J z_{1}$ and $J>0$. The free energy in Eq. (18), in the absence of magnetism, reduces to the standard case of order-disorder, but one of the species is twice degenerate. When magnetism is taken into account, model (18) exhibits two phase transitions respectively associated with the order parameters $\eta$ and $m$. We denote the respective transition temperatures by $T_{\eta}^{*}$ and $T_{m}^{*}$. Since we are interested in the case of $T_{m}^{*}<T_{\eta}^{*}$, the model parameters must be taken so that $\left(1+K^{*}\right)>J_{2}^{*}>0$.

The equilibrium temperature dependence of the order parameters was obtained from direct minimization of the func- 

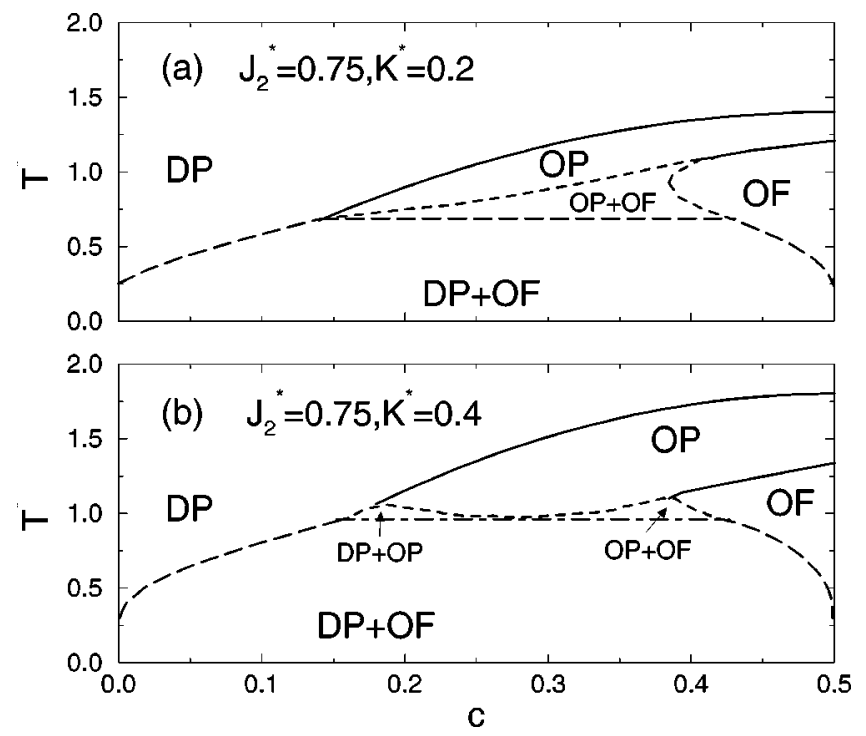

FIG. 4. Mean-field $c-T^{*}$ diagrams for two different sets of values of model parameters $J_{2}^{*}$ and $K^{*}$, indicating the disorderedparamagnetic phase (DP), the ordered-paramagnetic phase (OP), the ordered-ferromagnetic phase $(\mathrm{OF})$, and the coexistence regions.

tion (18). In Fig. 3 we show the $\mu^{*}-T^{*}$ section of the phase diagram for different values of $J_{2}^{*}=0.60,0.75$, and $K^{*}$ $=0.0,0.20$, and 0.40. Three different phases may appear: the disordered-paramagnetic (DP) phase with $\eta=0$ and $m$ $=0$, the ordered-paramagnetic (OP) phase with $\eta \neq 0$ and $m=0$, and the ordered-ferromagnetic (OF) phase with $\eta$ $\neq 0$ and $m \neq 0$. Both parameters $J_{2}^{*}$ and $K^{*}$ have the effect of increasing the stability of the ordered (OP and $\mathrm{OF}$ ) phases. Continuous lines stand for second-order phase transitions, whereas the dashed ones stand for discontinuous phase transitions. The intersection between the three interphases corresponds to a bicritical point in cases (a) and (b), whereas for (c) and (d) it corresponds to a triple point. The DP-OF transition is always first order, whereas the other two OP-OF and DP-OP may be second or first order. When the transition is first order, a phase separation shows up in the $c-T^{*}$ section. This is illustrated in Fig. 4 for cases (b) and (c) corresponding to the previous picture (Fig. 3).

In both cases of Fig. 4 a phase separation between a nonmagnetic (paramagnetic) and a ferromagnetic phase exists. At low temperatures the coexisting phases $(\mathrm{DP}+\mathrm{OF})$ are also different in their atomic ordered structure, whereas at moderate temperatures $(\mathrm{OP}+\mathrm{OF})$ both exhibit the same atomic structure. In addition, for case (b) (a larger value of $\left.K^{*}\right)$ a phase separation (DP+OP) between two nonmagnetic phases appears. In this case, there exists a line of triple points (horizontal dot-dashed line). In Fig. 5 we show the corresponding temperature behavior of the order parameters $\eta$ and $m$ for different values of the composition $c$. This information is obtained from the calculations presented in Fig. 4 taking into account the fact that in the phase separation region the system is heterogeneous and that at constant concentration both the characteristics and the amount of the coexisting phases change with temperature. It is noticeable that in both cases the two order parameters ( $\eta$ and $m$ ) exhibit an
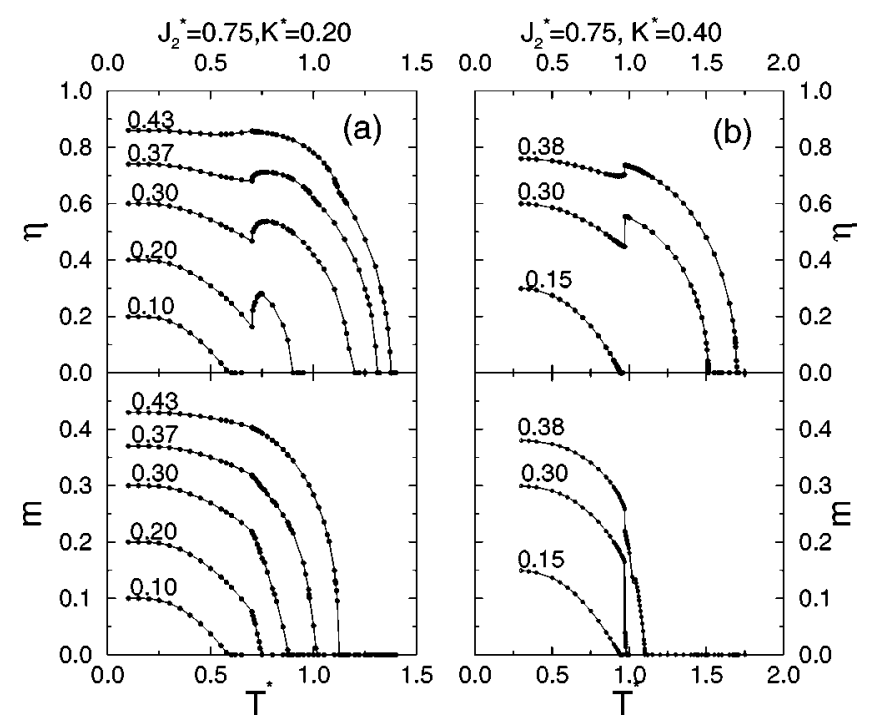

FIG. 5. Behavior of the order parameters $\eta$ and $m$ with temperature for different values of $c$ corresponding to the same two cases as in Fig. 4.

anomaly at a given temperature, (a) $T^{*} \sim 0.70$ and (b) $T^{*}$ $\sim 0.97$. These temperatures correspond to the bicritical and the triple points discussed in Fig. 3. When crossing the triple point line [case (b)], the anomaly is accompanied by a discontinuity in the order parameters.

\section{MONTE CARLO SIMULATION}

Monte Carlo simulations of model (10) have been performed in order to study the role of fluctuations. Starting from an initial (arbitrary) configuration, the subsequent microscopic configurations are generated by using the standard Metropolis algorithm. We have focused on two cases. First, we concentrated on the stoichiometric alloy $(c=0.5)$ for different values of the parameters $K^{*}$ and $J_{2}^{*}$. Secondly, we fixed $K^{*}=0.4$ and $J_{2}^{*}=0.6$ and studied the phase diagram as a function of $c$ and $T^{*}$.

\section{A. Simulation details}

The main results were obtained on a simple cubic lattice of size $L=16(N=L \times L \times L=4096)$. Moreover, a certain number of simulations with $L=24$ and $L=32$ were also carried out in order to study finite-size effects and to obtain illustrative real space snapshots of the system. Energy and order parameter fluctuations are measured according to the following definitions:

$$
\begin{gathered}
\mathcal{C}=\frac{1}{N T^{* 2}}\left(\left\langle H^{* 2}\right\rangle-\left\langle H^{*}\right\rangle^{2}\right), \\
\chi_{\eta}=\frac{1}{T^{*}}\left(\left\langle\eta^{2}\right\rangle-\langle\eta\rangle^{2}\right),
\end{gathered}
$$




$$
\chi_{m}=\frac{1}{T^{*}}\left(\left\langle m^{2}\right\rangle-\langle m\rangle^{2}\right)
$$

The angular brackets stand for Monte Carlo (MC) averages, performed over a large number of uncorrelated configurations after the equilibration of the system. In order to find the phase diagram, the transition lines were located from the positions of the peaks of the above quantities. In many cases equilibration was checked by testing the fluctuationdissipation theorem, i.e.:

$$
\mathcal{C}=\frac{1}{N} \frac{d\left\langle H^{*}\right\rangle}{d T^{*}} .
$$

Two kinds of numerical simulation experiments have been performed.

(1) Grand canonical simulations. The simulations in the grand canonical ensemble have the advantage of allowing faster equilibration. The alloy concentration is not fixed and an additional term taking into account the effect of the chemical potential difference between both species is needed in this case. Formally, this is done by a Legendre transformation of the Hamiltonian (10). This yields

$$
\begin{aligned}
\mathcal{H}^{*}= & \sum_{i j}^{\mathrm{NN}} \sigma_{i} \sigma_{j}-K^{*} \sum_{i j}^{\mathrm{NNN}} \sigma_{i} \sigma_{j} \\
& -J_{2}^{*} \sum_{i j}^{\mathrm{NNN}} \frac{1-\sigma_{i}}{2} \frac{1-\sigma_{j}}{2} S_{i} S_{j}+\mu^{*} \sum_{i=1}^{N} \sigma_{i} .
\end{aligned}
$$

Starting from an (arbitrary) initial configuration, the system at constant $T^{*}$ and $\mu^{*}$ evolves toward equilibrium by means of Glauber excitations proposed in both variables, $\sigma_{i}$ and $S_{i}$ independently. The unit of time MCS (a Monte Carlo step) is defined as $N$ independent proposals of each kind of flip on a randomly selected lattice site. Typically the averages are performed over 1500 configurations, taken every $20 \mathrm{MCS}$ 's and discarding the initial $5000 \mathrm{MCS}$ 's for equilibration. The regions of phase separation correspond to unreachable regions in the $c-T^{*}$ phase diagram.

(2) Canonical simulations. In these simulations the Glauber excitations are proposed in the magnetic variable $S_{i}$ only. In order to preserve the alloy composition $c$, the variables $\sigma_{i}$ evolve according to Kawasaki exchange dynamics. The equilibration process is much slower in this case and the system may get trapped in metastable configurations. To get rid of such configurations, it is convenient to allow a certain fraction $(q)$ of exchanges between NNN atoms. A MCS is in this case defined as $N$ proposals of $S_{i}$ flips, $N(1-q)$ proposals of NN exchanges, and $N q$ proposals of NNN exchanges. We have studied the effect of different values of $q$ and found that $q \sim 0.2$ is enough to reach equilibrium in a reasonable time. Typically averages are performed over 3500 configurations, taken every $50 \mathrm{MCS}$ 's, after discarding the first 25000 MCS's for equilibration. In the region of phase separation the simulated system evolves to an inhomogeneous "slab" configuration with a flat interface. Because of finitesize effects, the energy of such configurations is very much dominated by the interfacial energy and should be carefully

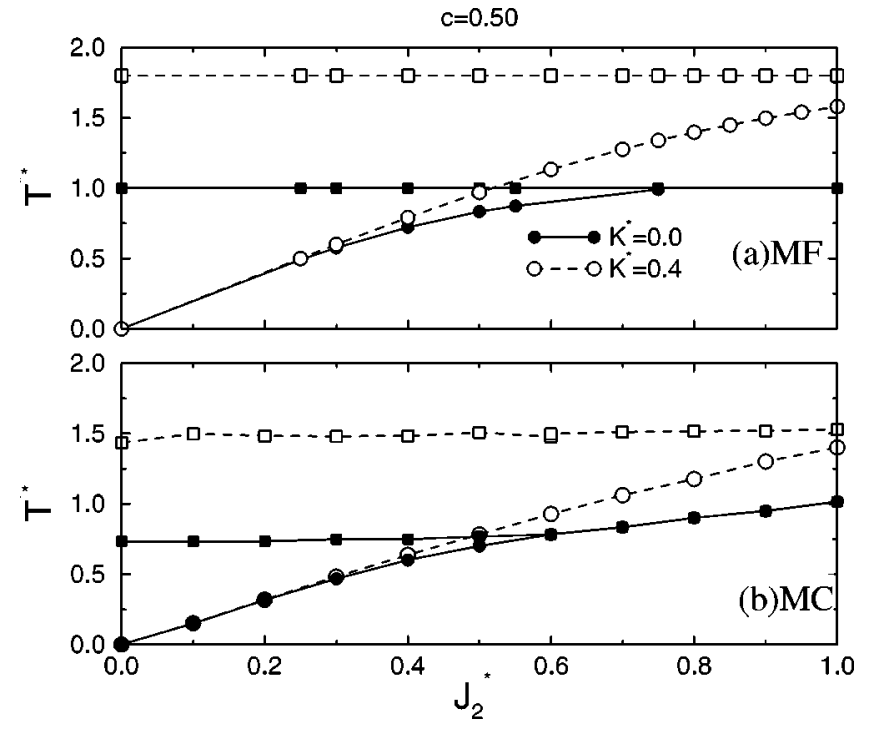

FIG. 6. Dependence of the transition temperatures $T_{\eta}^{*}(\square, \boldsymbol{\square})$ and $T_{m}^{*}(\bigcirc, \bigcirc)$ on $J_{2}^{*}$ for the stoichiometric compound $(c=0.5)$, from mean-field calculations (a) and Monte Carlo simulations (b). Open symbols correspond to $K^{*}=0.4$ and filled symbols to $K^{*}$ $=0.0$.

analyzed. In spite of the long times needed to get reliable results, the simulations in the canonical ensemble are very useful here since they provide information concerning the structure of the domains in the coexistence region.

\section{B. Monte Carlo results}

We start by presenting the transition temperatures as a function of the model parameters for the case of the stoichiometric alloy $c=0.5$. This is shown in the lower part of Fig. 6(b). A comparative look at both mean-field (a) and MC results (b) reveals that both solutions render the same qualitative behavior. The fluctuations (taken into account in the Monte Carlo solution) have the effect of increasing the stability of the disordered, paramagnetic phases so that the overall transition temperatures are lower than in the meanfield solution.

Figure 7 shows the $\mu^{*}-T^{*}$ section of the phase diagram, drawn from the grand canonical simulations, with $L$

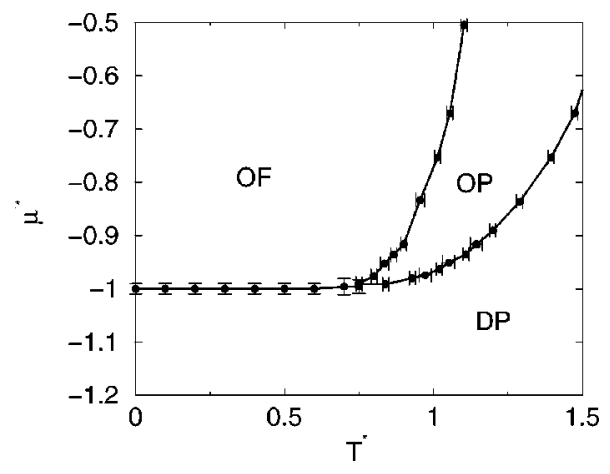

FIG. 7. Phase diagram $\mu^{*}-T^{*}$ for $J_{2}^{*}=0.60$ and $K^{*}=0.40 \mathrm{ob}-$ tained from Monte Carlo simulations in the grand canonical ensemble. Lines are guides to the eye. 


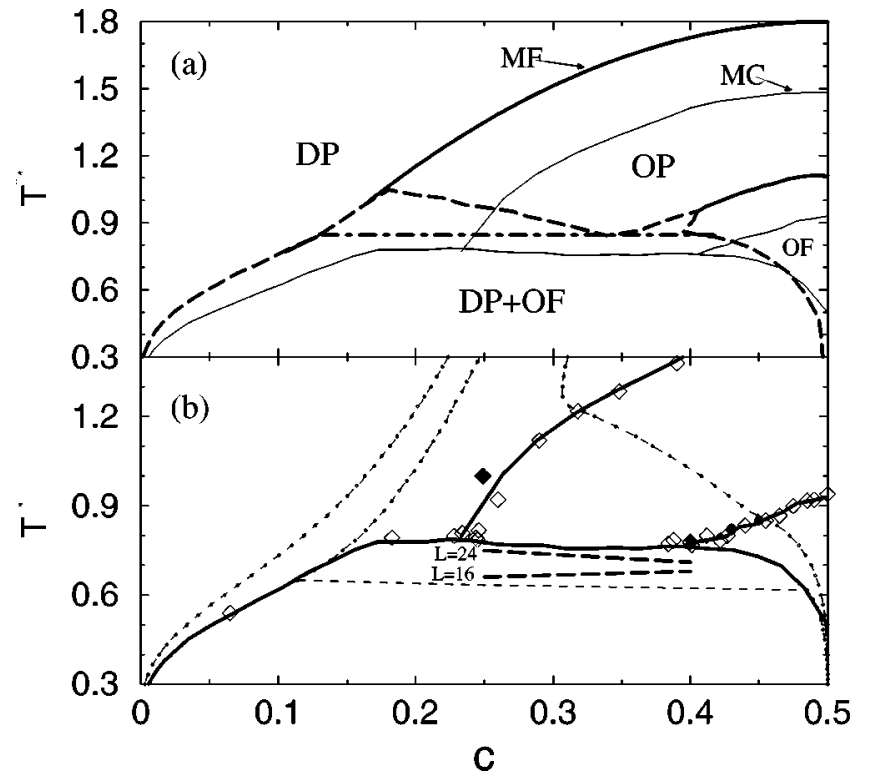

FIG. 8. Phase diagram $c-T^{*}$ for $J_{2}^{*}=0.60$ and $K^{*}=0.40 \mathrm{ob}-$ tained from mean-field calculations (a) and Monte Carlo simulations (b). Thick lines indicate the phase boundaries between the homogeneous phases and the coexistence regions. The thin lines with dots correspond to grand canonical MC runs at constant chemical potential; diamonds indicate the positions of the specific heat peaks from grand canonical (open diamonds) and canonical (black diamonds) simulations. Thick dashed lines in (b) are estimations of the coexistence region boundary from canonical simulations with $L=16$ and $L=24$. The continuous thin lines in (a) indicate the Monte Carlo phase boundaries for comparison.

$=16, J_{2}^{*}=0.6$, and $K^{*}=0.4$. We notice that the model parameters are those of Fig. 3(d). It follows that both numerical simulations and mean-field techniques render the same qualitative phase diagram. We remark only that there is smearing out of the reentrant (OP) phase in the MC solution, due to the fluctuations. The available MC data do not allow for a conclusive determination of the nature (first or second order) of the transitions.

In order to compare data with experiment, a study of the $c-T^{*}$ section of the phase diagram is essential. It turns out to be a tough task because of the finite-size effects. In particular, to definitively resolve the coexistence region, one needs to use very large linear system sizes.

Figure 8 shows the $c-T^{*}$ phase diagram corresponding to $J_{2}^{*}=0.6$ and $K^{*}=0.4$. In Fig. 8(a) we simultaneously show the mean-field and MC solutions. One observes that the main trends of both phase diagrams are the same. For practical reasons, we show the $\mathrm{MC}$ solution in more detail in Fig. 8(b). The phase transition lines and the limits of the coexistence region have been located from the peaks observed in the specific heat $\mathcal{C}$. This criterion has been followed in both the grand canonical (open diamonds) and canonical (black diamonds) simulations. In the grand canonical simulations the coexistence region is revealed by unreachable zones in the $\langle c\rangle-T^{*}$ diagram accompanied by flat steps in the curves of constant $\mu^{*}$ (three examples are depicted by small dots joined by a thin line).

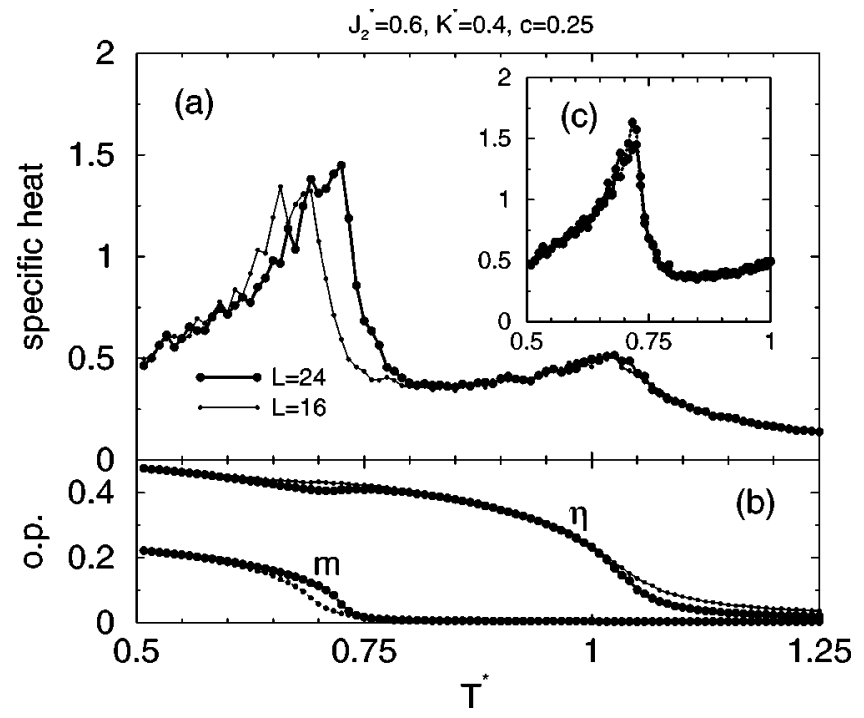

FIG. 9. Specific heat $\mathcal{C}$ and order parameters $m$ and $\eta$ as a function of temperature $T^{*}$ for $J_{2}^{*}=0.6, K^{*}=0.4$, and $c=0.25$ obtained from canonical MC simulations. Data for $L=16$ and $L$ $=24$ are shown with thin and thick lines, respectively. The inset (c) shows the specific heat computed from fluctuations (continuous line) as well as from the derivative of the average energy (dashed line).

When comparing the results corresponding to the same system size obtained from both the canonical and the grand canonical simulations we see that in the former the coexistence line occurs at lower temperatures. This is due to finitesize effects which have a strong influence on the stabilization of the mixed phase configurations. In this sense we have checked that when the system size is increased this effect is corrected and the phase separation occurs at higher temperatures. To illustrate this, we have plotted in Fig. 8 (with thick dashed lines) the upper part of the coexistence line obtained from canonical simulations, for two different values of the system size ( $L=16$ and $L=24)$, as indicated.

The same effect appears when studying the specific heat. In Fig. 9 we show the temperature behavior of the specific heat $\mathcal{C}$ (a) together with the order parameters $m$ and $\eta$ (b) for $J_{2}^{*}=0.6, K^{*}=0.4$, and $c=0.25$ as obtained from the canonical MC simulations. Data shown correspond to $L=16$

(a) DP

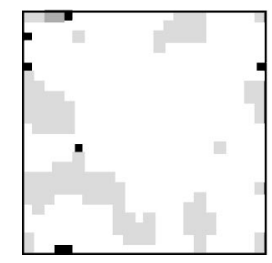

FIG. 10. Snapshots of the system configuration corresponding to the three different phases: (a) Disordered-paramagnetic (DP) phase at $T^{*}=1.2$ and $c=0.2$, (b) ordered-paramagnetic (OP) phase at $T^{*}=1.0$ and $c=0.32$, and (c) ordered-ferromagnetic (OF) phase at $T^{*}=0.79$ and $c=0.45$. The shading identifies the different phases locally according to the short-range order parameters as explained in the text. 
(a)

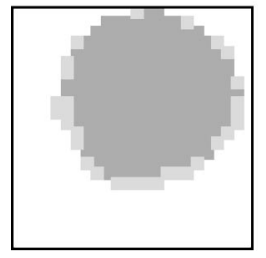

(b)

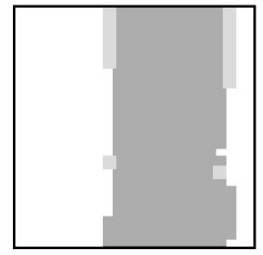

(c)

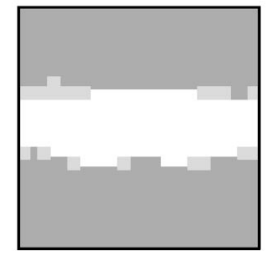

(d)

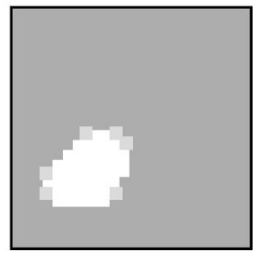

FIG. 11. Snapshots of the system configuration in the coexistence region for $T^{*}=0.5$ and $c$ $=0.1$ (a), $c=0.2$ (b), $c=0.3$ (c), and $c=0.45$ (d). and $L=24$. Note that the peak corresponding to the phase separation shows a much larger dependence on $L$ than the peak corresponding to the order-disorder transition. The inset (c) shows the specific heat computed from the energy fluctuations [Eq. (19)] and from the derivative of the average energy [Eq. (22)]. The agreement ensures that the equilibration times considered are long enough.

In spite of the difficulties described above, which certainly hinder the location of the boundaries, the phase diagram presented in Fig. 8 is essentially similar to that obtained experimentally (see Fig. 1) at least at moderate and low temperatures. The lack of resolution in the results makes it impossible to conclude whether or not the MC results give a line of triple points as occurs in the mean-field solution (lower part of 4). Unfortunately, the existing experimental data do not provide information on this point. We suggest that more experiments are needed. Provided that the experimental phase diagram is sufficiently well resolved, fine tuning of the parameters $J_{2}^{*}$ and $K^{*}$ (even $J_{1}^{*}$ ) would allow the matching of more details.

In addition to the determination of the phase diagram and the fluctuations, the MC simulations can provide real space snapshots of the system configuration. Figure 10 shows a two-dimensional section of the simulated system with $L$ $=24$ for different homogeneous equilibrium phases corresponding to the phase diagram in Fig. 8. Case (a) corresponds to the DP phase with $c=0.2$ and $T^{*}=1.2$, (b) to the OP phase with $c=0.32$ and $T^{*}=1.0$, and (c) to the OF phase with $c=0.45$ and $T^{*}=0.79$. The assignment of the different shading has been done by measuring the short-range order parameters in a cell of size $5 \times 5 \times 5$ centered at each lattice site of a certain two-dimensional horizontal cut of the original system. When the values of the local magnetization $m$ and/or local order parameter $\eta$ are above 0.2 the corresponding lattice site is considered to belong to a ferromagnetic and/or to an atomically ordered phase. White, light gray, and dark gray indicate DP, OP, and OF regions. Black corresponds to local disordered ferromagnetic regions which do not correspond to any stable phase. These appear because the fluctuations become both more probable and important with increasing temperature in the homogeneous phases. Actually, the three snapshots correspond to a time evolution of 2 $\times 10^{5}$ MCS's, when the average values of the long-range order parameters are perfectly equilibrated. Thus, the curved interfaces reveal that the fluctuations evolve with time and appear and disappear very quickly.

In Fig. 11 we show snapshots of the system configuration inside the coexistence region. The four pictures correspond to $T^{*}=0.5$ and to different values of the composition: (a) $c=0.1$, (b) $c=0.2$, (c) $c=0.3$, and (d) $c=0.45$. Note that for low concentration of the magnetic component (a), the OF phase consists of ferromagnetic bubbles inside the DP matrix as expected. For larger values of $c$ the ferromagnetic bubbles transform into rods or slabs (b). This is an artifact of finitesize effects that makes the system decrease its interfacial energy by taking advantage of the periodic boundary conditions. Cases (c) and (d) are symmetric to (b) and (a) respectively. Given the large value of $c$, the matrix is ferromagnetic and the domains paramagnetic.

It is known that the shape and size of the magnetic bubbles embedded into the nonmagnetic matrix is crucial for the occurrence of magnetoresistance. In the light of the present results, we believe the present model is suitable for determining the optimum characteristics of such domains. Along these lines, a study of kinetics of domain growth after quenches from high temperature should supply useful information. This will be the subject of future work.

\section{CONCLUSION}

By using a simple lattice model we have shown that the magnetism of an ordered alloy may give rise to a low temperature phase separation between a ferromagnetic phase and a paramagnetic phase. The existence of this mixed phase is relevant in relation to the occurrence of phenomena such as superparamagnetism and magnetoresistance.

This study was motivated by the behavior observed in $\mathrm{Cu}_{3-x} \mathrm{AlMn}_{x}$. Nevertheless, the strategy followed in the construction of the model should apply to other alloys, in particular, to those in which ferromagnetism is induced by configurational ordering of the magnetic atoms, as occurs in $\mathrm{Cu}_{3-x} \mathrm{AlMn}_{x}$. Our main conclusion is that this interplay between the two kinds of ordering is enough to produce the magnetic phase separation. We should mention that other effects such as elasticity due to the different atomic size of the elements may affect the final phase diagram. In spite of this and in view of the present results it is clear that the model captures the essential ingredients and makes it an appropriate starting point for future dynamical studies of the kinetics of formation of the mixed phase after a suitable thermal quench.

\section{ACKNOWLEDGMENTS}

We acknowledge fruitful discussions with Antoni Planes. The authors also acknowledge financial support from CICyT Project No. MAT98-0315. J.M. acknowledges financial support from Direcció General de Recerca (Catalonia). 
${ }^{1}$ K. Ullakko, P.T. Jakovenko, and V.G. Gavriljuk, Proc. SPIE 2715, 42 (1996).

${ }^{2}$ T.V. Yefimova, V.V. Kokorin, V.V. Polotnyuk, and A.D. Shevchenko, Phys. Met. Metallogr. 64, 189 (1987).

${ }^{3}$ L. Yiping, A. Murthy, G.C. Hadjipanayis, and H. Wan, Phys. Rev. B 54, 3033 (1996).

${ }^{4}$ P.J. Webster, Contemp. Phys. 10, 559 (1969).

${ }^{5}$ P.J. Webster, K.R.A. Ziebeck, S.L. Town, and M.S. Peak, Philos. Mag. B 49, 295 (1984).

${ }^{6}$ J.S. Robinson, S.J. Kennedy, and R. Street, J. Phys.: Condens. Matter 9, 1877 (1997).

${ }^{7}$ S. Plogmann, T. Schlathölter, J. Braun, M. Neumann, Yu.M. Yarmonshenko, M.V. Yablonskikh, E.I. Shreder, E.Z. Kurmaev, A. Wrona, and A. Slebarski, Phys. Rev. B 60, 6428 (1999).

${ }^{8}$ Y. Ishikawa, Physica B \& C 91B, 130 (1977).

${ }^{9}$ A. Planes, E. Obradó, A. Gonzàlez-Comas, and Ll. Mañosa, Phys. Rev. Lett. 79, 3926 (1997), and references therein.

${ }^{10}$ T. Castán, E. Vives, and P.-A. Lindgård, Phys. Rev. B 60, 7071 (1999), and references therein.

${ }^{11}$ J. Soltys, Phys. Status Solidi A 63, 401 (1981).

${ }^{12}$ M. Bouchrad and G. Thomas, Acta Metall. 23, 1485 (1975).

${ }^{13}$ M. Prado, F. Sade, and F. Lovey, Scr. Metall. Mater. 28, 545 (1993).

${ }^{14}$ E. Obrado, Ll. Mañosa, and A. Planes, Phys. Rev. B 56, 20 (1997).

${ }^{15}$ E. Obrado, C. Frontera, L1. Mañosa, and A. Planes, Phys. Rev. B 58, 14245 (1998).

${ }^{16}$ E. Obrado, A. Planes, and B. Martinez, Phys. Rev. B 59, 11450
(1999).

${ }^{17}$ E. Obrado, E. Vives, and A. Planes, Phys. Rev. B 59, 13901 (1999).

${ }^{18}$ A.J. Bradley and J.W. Rodgers, Proc. R. Soc. London, Ser. A 144, 340 (1934).

${ }^{19}$ L. Delaey, in Materials Science and Technology, edited by P. Haasen (VCH, Weinheim, 1991), Vol. 5, p. 339.

${ }^{20}$ K. Ullakko, J.K. Huang, C. Kantner, R.C. O’Handley, and V.V. Kokorin, Appl. Phys. Lett. 69, 1966 (1996).

${ }^{21}$ A.A. Likhachev and K. Ullakko (unpublished).

${ }^{22}$ In the case of $\mathrm{Ni}_{2} \mathrm{MnGa}$, the cubic $L 2_{1}$ phase that transforms martensitically is ferromagnetic.

${ }^{23}$ J. Marcos, A. Planes, Ll. Mañosa, A. Labarta, and B.J. Hattink, (unpublished).

${ }^{24}$ R. Kainuma, N. Satoh, X.J. Liu, I. Ohnuma, and K. Ishida, J. Alloys Compd. 266, 191 (1998).

${ }^{25}$ E. Obradó, Ph.D. thesis, Universitat de Barcelona, 1999.

${ }^{26}$ K. Nicolaus, Ph.D. thesis, Technische Universität München, 2000.

${ }^{27}$ J.L. Murray, in Binary Alloy Phase Diagrams, edited by T.B. Massalski (American Society for Metals, Metals Park, OH, 1986), Vol. 1, p. 103.

${ }^{28}$ D.R.F. West and D. Lloyd Thomas, J. Inst. Met. 85, 97 (1956).

${ }^{29}$ G.B. Johnston and E.O. Hall, J. Phys. Chem. Solids 29, 201 (1968).

${ }^{30}$ K. Tajima, Y. Ishikawa, P.J. Webster, M.W. Stringfellow, D. Tochetti, and K.R.A. Ziebeck, J. Phys. Soc. Jpn. 43, 483 (1977).

${ }^{31}$ E. Vives, E. Obradó, and A. Planes, Physica B 275, 45 (2000). 